\title{
Detection of Cyber-attacks in Systems with Distributed Control based on Support Vector Regression
}

\author{
Dusan M. Nedeljkovic, Zivana B. Jakovljevic, Member, IEEE, \\ Zoran Dj. Miljkovic, and Miroslav Pajic, Senior Member, IEEE
}

\begin{abstract}
Concept of Industry 4.0 and implementation of Cyber Physical Systems (CPS) and Internet of Things (IoT) in industrial plants are changing the way we manufacture. Introduction of industrial IoT leads to ubiquitous communication (usually wireless) between devices in industrial control systems, thus introducing numerous security concerns and opening up wide space for potential malicious threats and attacks. As a consequence of various cyber-attacks, fatal failures can occur on system parts or the system as a whole. Therefore, security mechanisms must be developed to provide sufficient resilience to cyber-attacks and keep the system safe and protected. In this paper we present a method for detection of attacks on sensor signals, based on $\varepsilon$ insensitive support vector regression ( $\varepsilon$-SVR). The method is implemented on publicly available data obtained from Secure Water Treatment (SWaT) testbed as well as on a real-world continuous time controlled electro-pneumatic positioning system. In both cases, the method successfully detected all considered attacks (without false positives).
\end{abstract}

Keywords - Cyber Physical Systems, Cyber Security, Industrial Control Systems, Industrial Internet of Things, Support Vector Regression.

\section{INTRODUCTION}

$\mathrm{I}$ MPLEMENTATION of Internet of Things (IoT) [1] in manufacturing environment leads to a new concept of manufacturing known as Industry 4.0 [2]. Within this concept elements of manufacturing systems are created in the form of Cyber Physical Systems (CPS) [3] that integrate a physical process and its cyber representation through real time interaction. In industrial environment, CPSs are implemented at various hierarchy levels (control device,

Paper received May 22, 2020; revised July 18, 2020; accepted July 31, 2020. Date of publication December 25, 2020. The associate editor coordinating the review of this manuscript and approving it for publication was Prof. Branimir Reljin.

This paper is revised and expanded version of the paper presented at the 27th Telecommunications Forum TELFOR 2019 [13].

This research was supported by the Science Fund of the Republic of Serbia, grant No. 6523109, AI- MISSION4.0, 2020-2022.

The research in this paper was supported by the Ministry of Education, Science and Technological Development of the Serbian Government, 451-03-68/2020-14/200105.

Dusan M. Nedeljkovic, Zivana B. Jakovljevic, and Zoran Dj. Miljkovic are with the University of Belgrade, Faculty of Mechanical Engineering, Department for Production Engineering, Kraljice Marije 16, 11120 Belgrade, Serbia (e-mail: dnedeljkovic@mas.bg.ac.rs; zjakovljevic@mas.bg.ac.rs; zmiljkovic@mas.bg.ac.rs).

Miroslav Pajic is with the Department of Electrical and Computer Engineering, Duke University, Durham, NC 27708 USA (email: miroslav.pajic@duke.edu). station, work center, enterprise...) as systems of systems. The core of CPS in Industry 4.0 represent smart devices (sensors, actuators, machines...) in which physical devices are augmented with computation and communication capabilities. Consequently, within Industry 4.0, control tasks are distributed over smart devices that are capable of local control, autonomous decision making, and information exchange. In such systems, ubiquitous communication (usually wireless) is required. This leads to a large number of objects involved in the network, which represents a vast area for threats and malicious cyberattacks. These attacks often lead to anomalies and serious consequences which can completely disable system functioning. To solve these security issues and keep the system under normal conditions, defense techniques with high-level protection must be developed. In most cases, timely detection and response to cyber-attacks can help to minimize their potential impact. Since attacks tend to be stealthy and do not show immediately a physical effect on the system behavior, their detection represents a challenging task.

We focus on deception attacks characterized by the injection of false data into network communication links between control system components [4]. Generally, in industrial applications discrete event and continuous time systems are present. Due to the inherent differences in these systems the approaches to their modeling and control are different. Consequently, the design of the attacks and corresponding mechanisms for their detection and system resilience improvement require implementation of different techniques [4] - [6]. In this research work, we focus on distributed continuous time controlled systems and on the attacks on the vulnerable communication links between controller and remote sensors/actuators in these systems (Fig. 1).

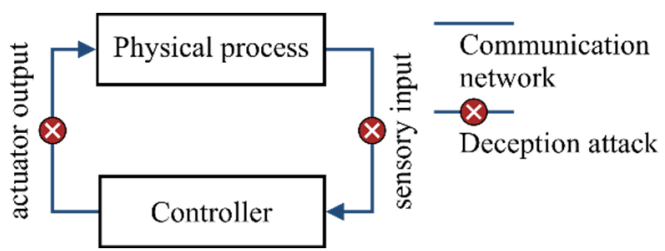

Fig. 1. Vulnerable communication links between controller and remote sensors/actuators in continuous time controlled systems [4].

Depending on the resources they utilize, cyber-attacks detection techniques can be data-centric (use collected data) 
and design-centric (use an analytical model of the process and its control algorithms) [7]. An example of designcentric approach that utilizes a considered CPS model for attack detection and identification is presented in [8]. Nevertheless, due to the complexity of the controlled processes, in a large number of cases the valid analytical model is not at disposal. Thus, a number of data-centric detection techniques were developed to deal with this issue; they are based on convolutional neural networks [9], deep neural networks and one-class support vector machines [10], autoregression modeling, and control limits [11].

The method we present in this paper is based on $\varepsilon$ insensitive support vector regression ( $\varepsilon$-SVR) and it belongs to a group of data-centric techniques. In our previous work [5] we have explored the possibilities of $\varepsilon$ SVR based sensory signal cyber-attack detection on the publicly available dataset obtained from a scaled down water treatment plant. The method has shown great potential; nevertheless, its application was carried out offline. The computational complexity of $\varepsilon$-SVR raises the issue of the real-time online applicability and generalization performances of the proposed method.

In this work, we implement our method on both the publicly available data obtained from Secure Water Treatment (SWaT) testbed [12] and on the real-world installation - an electro-pneumatic positioning system with a control system distributed over smart devices. As will be presented in the sequel, the method has shown offline and real-time applicability with high generalization capabilities demonstrated on a number of cyber-attacks.

The remainder of the paper is structured as follows. Section 2 refers to the developed method for signal attacks detection. In Section 3 we represent implementation and experimental evaluation of the proposed method. Finally, in Section 4 we provide conclusions and future work guidelines.

\section{Signal AtTACK DETECTION METHOD}

The method for signal attack detection consists of two phases: 1) offline training phase in which $\varepsilon$-SVR model of the data communicated between remote devices is generated, and 2) online attack detection (Fig. 2). In the offline phase, using signal (time series) $x_{1}, \ldots, x_{k}, \ldots, x_{n}$ acquired under normal operating conditions, $\varepsilon$-SVR model for the estimation of the signal under normal system operation (without attack) is generated.

Namely, using our approach [5], [13], a current value of the signal $x_{i}$ is estimated from the buffer of previous $k$ values $x_{i-k}, \ldots, x_{i-1}$. Thus, $\varepsilon$-SVR training set has the following form:

$$
\begin{gathered}
\left(\mathbf{x}_{\mathbf{i}}, y_{i}\right) \in\left(\left[x_{1}, \ldots, x_{k}\right], x_{k+1}\right), \\
\left(\left[x_{2}, \ldots, x_{k+1}\right], x_{k+2}\right), \ldots,\left(\left[x_{n-k}, \ldots, x_{n-1}\right], x_{n}\right)
\end{gathered}
$$

where $\mathbf{x}_{\mathbf{i}}, i \in[k+1, n]$ denotes input variables vector, and $y_{i}$ represents the corresponding response value during $\varepsilon$-SVR training.

$\varepsilon$-SVR model is obtained in the form:

$$
\hat{x}_{i}=\sum_{n s}\left(\alpha_{j}-\alpha_{j}^{*}\right) K\left(\left[x_{i-k}, \ldots, x_{i-1}\right], \mathbf{x}_{\mathbf{j}}\right)+b
$$

where $\hat{x}_{i}$ represents the predicted value of $x_{i}, \mathbf{x}_{\mathbf{j}}$ are support vectors, i.e., input variables vectors for which the Lagrange multipliers $\alpha_{j}$ and $\alpha_{j}^{*}$ in $\varepsilon$-SVR optimization problem are nonzero $^{1}, K$ is a kernel function that defines inner product in hyperspace and $b$ is a bias term obtained during $\varepsilon$-SVR training.

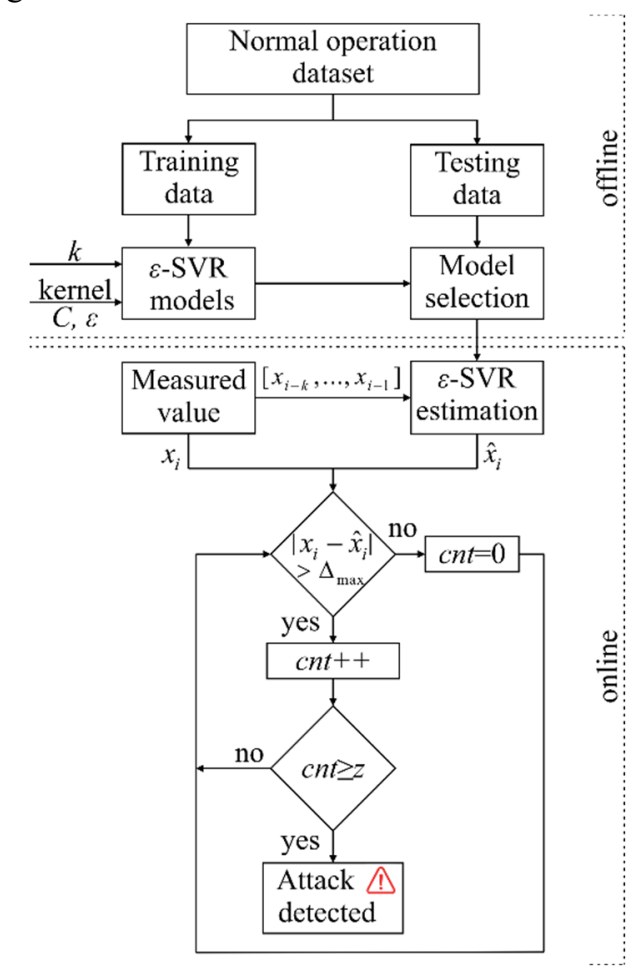

Fig. 2. Schematic diagram of the method for signal attacks detection.

A number of different kernel functions such as polynomial kernel, radial basis kernel, wavelet kernel, sigmoid kernel can be used in $\varepsilon$-SVR [15]. The kernel chosen for the application in this work is a radial basis function defined as follows:

$$
K\left(\mathbf{x}, \mathbf{x}_{\mathbf{j}}\right)=\exp \left\{-\gamma\left|\mathbf{x}-\mathbf{x}_{\mathbf{j}}\right|^{2}\right\}
$$

where $\gamma$ determines the width of the bell-shaped curve.

To get as good a model as possible, the buffer length $k$ and $\varepsilon$-SVR parameters ${ }^{1}(C, \varepsilon$ and radial basis function parameter $\gamma$ ) should be tuned. Each combination of abovementioned parameters determines one $\varepsilon$-SVR model. In our approach, the selection of the optimal model is based on two criteria: 1) the number of support vectors ( $n s)$, and 2) the model accuracy over the whole dataset (training and testing data). Reduction of the number of support vectors results in the simplicity of the model and a decrease of computational complexity that is crucial for the decrease of latency in a subsequent online application. However, too small number of support vectors can result in an inaccurate model. The second criterion is employed in order to evaluate the quality of the estimation. Including the whole dataset, we consider the number $\left(\Delta_{\text {out }}\right)$ of estimated values whose absolute errors 
with respect to response values exceeded the predefined threshold $m$. The threshold $m$ is defined as the mean absolute deviation between the real $x_{i}$ and the estimated values $\hat{x}_{i}$ over the whole dataset:

$$
m=100 \frac{1}{n} \sum_{i=k+1}^{n}\left|x_{i}-\hat{x}_{i}\right|
$$

After selecting the model through offline training, according to the defined criteria, online attack detection is performed based on the difference between the estimated and measured values. An attack is present if an absolute error between measured $x_{i}$ and estimated $\hat{x}_{i}$ actuator signal value exceeds the detection threshold $\left(\Delta_{\max }\right)$ consecutively for $z$ estimated values.

$$
\left|x_{i}-\hat{x}_{i}\right|>\Delta_{\max }
$$

\section{IMPLEMENTATION AND EXPERIMENTAL EVALUATION OF THE PROPOSED METHOD}

The method for detection of cyber-attacks is tested on the publicly available dataset generated on the SWaT testbed [12] as well as on an electro-pneumatic positioning system (DisEPP) developed at the Laboratory for Manufacturing Automation (LMA).

\section{A. Case Study 1 - Secure Water Treatment testbed}

The first case study refers to Secure Water Treatment (SWaT) testbed [12], a fully operational scaled-down water treatment plant created at the Singapore University of Technology and Design. It was built for investigating in the field of cyber security, especially to experimentally validate novel designs of defense techniques [16]. Besides, it is capable of producing 5 gallons of purified water per minute. The whole water treatment process is divided into 6 cooperating stages, marked with $\mathrm{P} 1-\mathrm{P} 6^{2}$. Each stage is controlled by an independent PLC (Programmable Logic Controller), where control actions are based on sensor signals. In SWaT, sensors are usually used to check the physical and chemical properties of water. Communications between sensors, actuators and PLCs in the plant are via wired or wireless links. All PLCs are connected to the SCADA (Supervisory Control and Data Acquisition) system to monitor the whole SWaT process.

The data collection process lasted 11 days, the SWaT system worked continuously 24 hours/day. Recorded data were obtained from the sensors and actuators contained in the testbed. For the first 7 days, data were generated every second based on the normal functioning of the system (without attacks). Diverse attacks were present during the last 4 days. A total of 41 attacks of various lasting and intensity have been created, whereby 36 attacks have a physical impact on the system. Depending on the location, all attacks can be divided into attacks that act on single/multiple points within single/multiple stages.

In the focus of this work are anomalies/attacks affecting the LIT301 sensor, a water level sensor on UF feed water tank, which is located in the third stage ${ }^{2,3}$. Attack on the LIT301 sensor signal can lead to underflow/overflow of the
UF feed water tank. This can further cause serious damages to the UF feed pump (P301) and other devices of the system. The proposed method for attacks detection was implemented in MATLAB and tested using LIT301 sensor signal data under normal operation (Fig. 3). Period of establishing a stable operating mode of the system was omitted from the dataset used for offline training. For the considered sensor LIT301, a stable operating mode means charging and discharging of the tank.

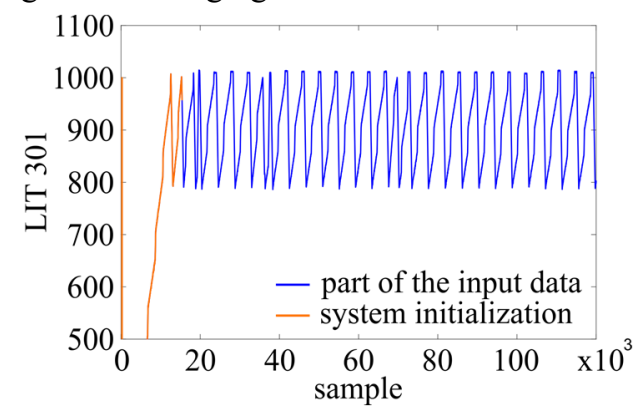

Fig. 3. Part of the input data under normal conditions.

Therefore, the first 15400 data records were removed, which resulted in a total of 481400 samples in the normal operation dataset. For $\varepsilon$-SVR training, $10 \%$ of normal operation data were utilized. For each combination of parameters values in the $\varepsilon$-SVR training, one unique model was generated. Through the model testing process, we have concluded that the error cost parameter $C$ and kernel parameter $\gamma$ do not have a significant influence on the model accuracy. Therefore, we have varied two parameters that have the main influence on the model: buffer size $k$ in the range of 2 to 10 and the $\varepsilon$ between 0.01 and 1 . The model with $k=2$ and $\varepsilon=1$ proved to be the best by both criteria ( 324 support vectors and $\Delta_{\text {out }}=0$ ). The selected model is employed for the detection of attacks intended for the online part of the proposed method and it was tested on 449919 records obtained during system performance under attack. The detection parameters are set to $z=2$ and $\Delta_{\max }=30$. Our method was able to effectively detect all five attacks on the LIT301 sensor, as shown in Fig. 4. Input data (LIT301 signal during attacks on the SWaT) and their predicted values are represented in blue and red line, respectively. Point of the attack is represented with a black dashed line, whereas the moment of attack detection is marked with a green $*$.

The group of detected attacks includes ${ }^{4}$ : (1) single stage single point attacks on LIT301 (attack 7 - Fig. 4a, attack 16 - Fig. 4b, attack 32 - Fig. 4d and attack 41 - Fig. 4e), and (2) multi stage single point attack 26 on LIT301 and raw water pump P101 (Fig. 4c).

Besides the attack on LIT301, our method also detected two attacks on adjacent devices which have affected the UF feed water tank level. Specifically, single stage multi point attack on raw water pumps P101 and P102 (attack 35 - Fig. 5a) and single stage single point attack on raw water tank level sensor LIT101 (attack 36 - Fig. 5b) were detected. It is worth noting that the proposed method detected attacks without false-positive results.

\footnotetext{
${ }^{4}$ Attacks on SWaT are labeled as in [16].
}

\footnotetext{
${ }^{2} \mathrm{SWaT}$ testbed processes overview is presented in [16].

${ }^{3}$ It should be noted that in [5] we have considered LIT101 sensor
} 


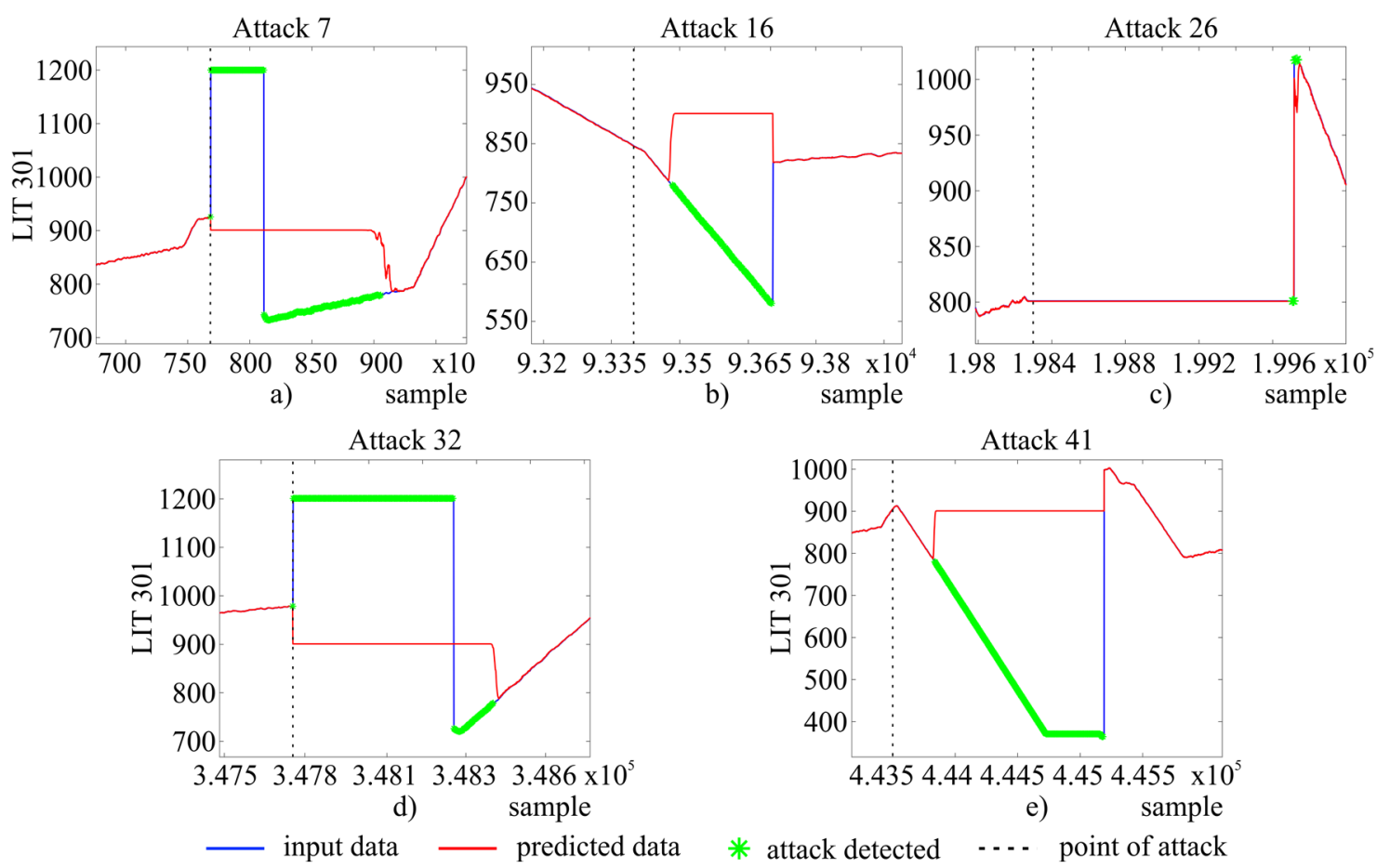

Fig. 4. Detected attacks on the LIT301; the details regarding the attacks can be found in [16].
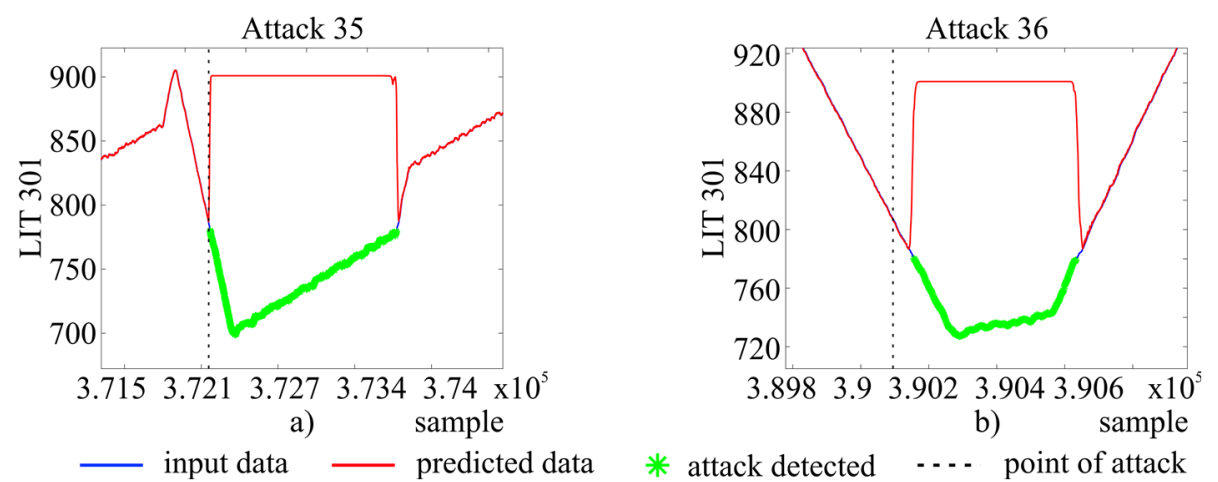

Fig. 5. Detected attacks on the adjacent devices; the details regarding the attacks can be found in [16].

\section{B. Case Study 2 - Electro-pneumatic positioning system}

To check the real-world applicability of the proposed method, we have implemented it on a custom made electropneumatic positioning system (DisEPP) with a control task distributed over smart devices. DisEPP is based on a smart actuator and smart sensor developed at LMA. A smart actuator consists of a linear rodless pneumatic cylinder SMC MY3B16-600 that is supplied by air through a mechanically controlled air pressure regulator AZ Pneumatica MREG 2-08 on one, and an electro-pneumatic air pressure regulator SMC ITV2050-33F2N3 on the other side (Fig. 6). Both regulators, through the air preparation unit are supplied with a constant pressure of 6 bar. The electro-pneumatic regulator transmits pressure (in the range 2-6 bar) whose intensity is proportional to the analog signal at its input (in the range $0-10 \mathrm{~V}$ ). On the other side of the cylinder, the mechanical regulator gives a constant pressure value of 4 bar. The piston movement is realized by the air pressure difference between the two sides of the piston.

In addition to electro-pneumatic components, a smart actuator contains a local controller $\left(\mathrm{LC}_{1}\right)-\mathrm{a}$ wireless node based on ARM Cortex-M3 running at $96 \mathrm{MHz}$ [17] augmented with an IEEE 802.15.4-compliant wireless transceiver Microchip MRF24J40MA [18].

In DisEPP, a control loop is closed using a linear encoder Balluff BML-S1B0-Q53G-M400-L0-KA05, positioned along the cylinder. The encoder is equipped with its own local controller - a wireless node $\left(\mathrm{LC}_{2}\right)$ based on the same devices as in actuator and it also has communication capabilities.

Cylinder stroke is $600 \mathrm{~mm}$, which corresponds to 60000 encoder pulses $(1 \mathrm{~mm}=100$ pulses $)$. End of cylinder on the mechanical air pressure regulator side is selected as the initial position, whereas a current position is determined from encoder signals.

A control task is distributed among $\mathrm{LC}_{1}$ and $\mathrm{LC}_{2}$ as follows. $\mathrm{LC}_{2}$ determines the position of the piston based on the pulses from $\mathrm{A}$ and $\mathrm{B}$ phases of the encoder. Furthermore, the desired position is set using $\mathrm{LC}_{2}$, and PID control is implemented in this node. Using PID (PID controller parameters were obtained experimentally), from current position of the piston, value in the range $[0,1]$ corresponding to the desired control signal of the actuator is obtained. This value is transmitted to $\mathrm{LC}_{1}$ using an IEEE 802.15.4 - compliant wireless transceiver. 


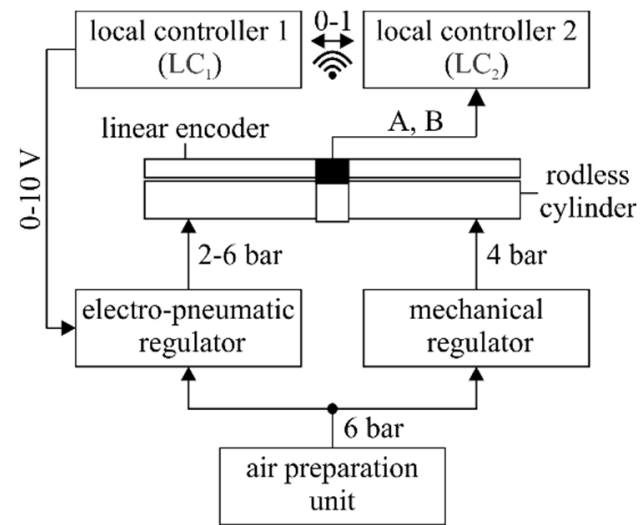

Fig. 6. Schematic diagram of experimental setup.

$\mathrm{LC}_{1}$ receives this value and converts it to the analog voltage in the range $0-10 \mathrm{~V}$ that is proportional to the air pressure necessary for the desired piston movement.

Wireless communication of signal from $\mathrm{LC}_{2}$ to $\mathrm{LC}_{1}$ represents the vulnerable point from cyber-attack point of view. To protect actuator from the potential attacks, we have implemented the method from Section II.

The first step during offline phase is to obtain a dataset that contains data that is communicated between nodes during normal system operation, i.e., without attacks. This dataset is obtained by acquisition of voltage between $\mathrm{LC}_{1}$ and the electro-pneumatic air pressure regulator using National Instruments Data Acquisition (DAQ) system; sampling rate is set to $100 \mathrm{~Hz}$. During data acquisition, after initialization (the first 1000 data records), the piston cyclically repeats 100 times the defined trajectory of 5 positions (expressed in $\mathrm{mm}$ ): 50, 400, 250, 400, and 100 . The data acquisition resulted with a total of 400,000 records representing the normal operation dataset (Fig. 7).

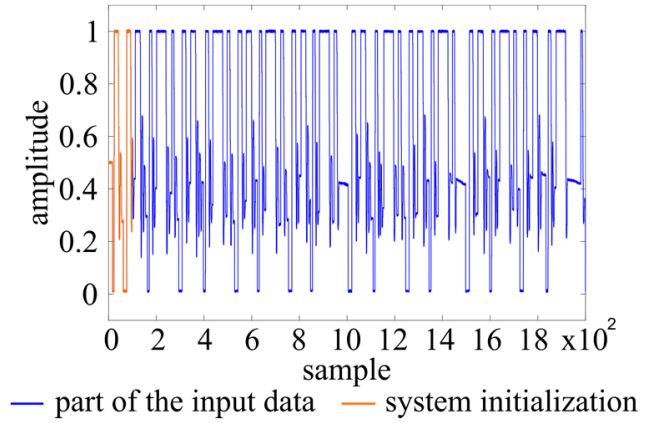

Fig. 7. DisEPP initialization and part of the data during normal system functioning.

For $\varepsilon$-SVR training, we employed $10 \%$ of dataset under normal conditions. $\varepsilon$-SVR models have been created using different combinations of $\varepsilon$-SVR parameters $(C, \varepsilon$ and radial basis function parameter $\gamma$ ) and buffer size $k$. Through the process of finding the optimal model, with the criteria of its accuracy and number of support vectors, we concluded that the error cost parameter $C$ and kernel parameter $\gamma$ did not have a significant influence, as in the case study 1 . The other two parameters were varied, buffer size $k$ from 2 to 10 and $\varepsilon$ in the range from 0.01 to 1 . The best model by criterion of the number of support vectors (4234) is obtained with parameters $\varepsilon=0.1$ and $k=2$; in this case $\Delta_{\text {out }}=13$. With the increase of parameter $\varepsilon$, the value of $\Delta_{\text {out }}$ decreases slightly, but the number of support vectors increases rapidly.
Therefore, the model with parameters $\varepsilon=0.1$ and $k=2$ is chosen as optimal.

Obtained $\varepsilon$-SVR model is implemented in $\mathrm{LC}_{1}$. Following the procedure from Fig. 2, the data received from $\mathrm{LC}_{2}$ are compared with data estimated using $\varepsilon$-SVR and based on the number of consecutive crossings of threshold the attack is detected. The number of the consecutive crossings in (5) before attack detection is set to $z=50$, whereas the value of the threshold $\Delta_{\max }=0.02$.

To test the actuator signal attack detection method on an experimental installation, a number of attacks with different types and duration have been created. The attack affects the input voltage of the electro-pneumatic controller, and accordingly the output pressure, which results in a changed piston path. All attacks aim to disrupt the piston in achieving the desired trajectory. In this paper we present three different attacks. Attack 1 increases the value of $x$ linearly, with the addition of a random number, as in (6).

$$
\begin{gathered}
x(i)=x(i)+0.00007 \cdot i+0.0005 \cdot \operatorname{rand}(), \\
i=1,2, \ldots, 400
\end{gathered}
$$

The consequence of the attack is a linear increase of pressure on the electro-pneumatic regulator to its maximum value, i.e. movement of the piston towards the initial position. Attack 2 immediately sets $x$ to 0 ; since the pressure on the electro-pneumatic controller is 0 , piston moves in direction from the initial position (7).

$$
x(i)=0, \quad i=1,2, \ldots, 500
$$

Attack 3 generates $x$ as a sine function (8) and thus interferes piston in reaching a defined position.

$$
x(i)=0.5+\sin (0.005 \cdot i), \quad i=1,2, \ldots, 1300
$$

All three signals with attacks, along with the predicted values are presented in Fig. 8. Proposed method was able to detect all three attacks on the system, as shown in Fig. 8. Moments when conditions (5) and $c n t \geq z$ were fulfilled, i.e., when the attack was detected, are marked with green *.

During system functioning, the method provides attack detection without false positives. It should be noted that to test the generalization properties of the proposed method, the method was tested not only for the trajectories used during generation of training data, but also for a number of different trajectories, such as 300/450/50/300/50, $150 / 350 / 450 \mathrm{~mm}$, etc. Furthermore, the application of $\varepsilon$ SVR did not generate any disturbances on the system performance - the system behaved the same as in the case without implementation of cyber-attack detection mechanism.

\section{CONCLUSION}

In this paper, we have presented and implemented a method for signal attack detection in continuous time controlled systems that is based on the prediction of signal value using $\varepsilon-S V R$. The method was evaluated on both the dataset obtained from SWaT testbed and on an electropneumatic positioning system. As presented in the paper, our method has proven to be effective in detecting attacks on the water level sensor LIT301 in SWaT testbed, as well as attacks on adjacent devices. Furthermore, our method is 
able to successfully detect cyber-attacks in the real-world application (an electro-pneumatic positioning system), without false positives and for different cylinder trajectories, thus presenting good generalization properties. The implementation of attack detection mechanism on a smart device (i.e., on $\mathrm{LC}_{1}$ attached to a smart cylinder) was able to detect the attacks in real-time and did not lead to the deterioration of the system performance. Nevertheless, the real-time applicability of the method can be constrained by the nature of sensory signals that directly influence the number of support vectors and consequently the computational cost of the attack detection method. It is possible that in some cases the performances of installed local controllers within smart devices would not enable real-time applicability and that the augmentation of their computational capabilities would be necessary. Our future research efforts will be directed to the development of an algorithm for automatic input parameter optimization and implementation of the method in more complex control systems.
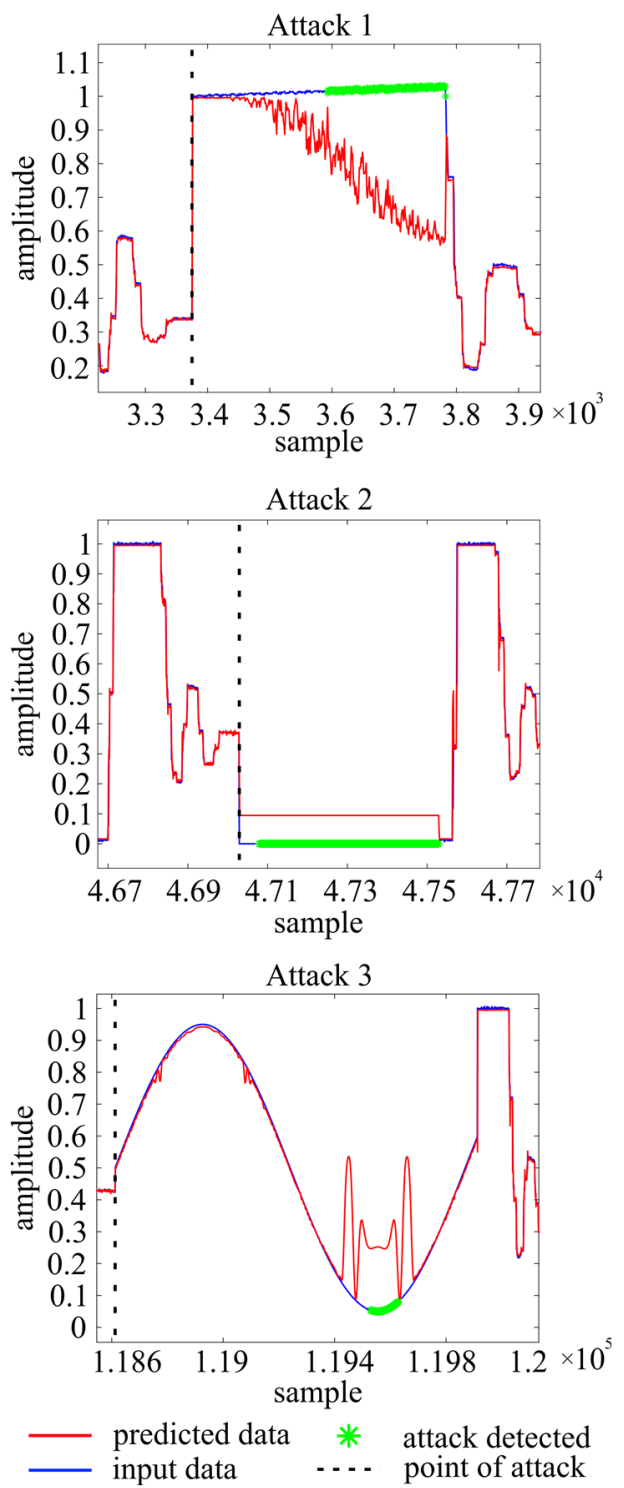

Fig. 8. Detected attacks.

\section{REFERENCES}

[1] L. Atzori, A. Iera, and G. Morabito, "The Internet of Things: A survey," Computer Networks, vol. 54, no. 15, pp. 2787-2805, Oct. 2010.

[2] H. Kagermann, W. Wahlster, and J. Helbig. Recommendations for implementing the strategic initiative INDUSTRIE 4.0, 2013. [Online]. Available: http://www.acatech.de

[3] L. Wang, M. Törngren, and M. Onori, "Current status and advancement of cyber-physical systems in manufacturing," Journal of Manufacturing Systems, vol. 37, no. 2, pp. 517-527, Oct. 2015.

[4] S. Mitrović, Z. Dimić, and Ž. Jakovljević, "Distributed control of manufacturing resources - security related issues," in Proceedings of MMA 2018 Conference, Sep. 2018, pp. 195-198.

[5] D. Nedeljković, Ž. Jakovljević, and Z. Miljković, "The detection of sensor signal attacks in industrial control systems," FME Transactions, vol. 48, no. 1, 2020.

[6] Z. Jakovljevic, V. Lesi, and M. Pajic, "Attacks on Distributed Sequential Control in Manufacturing Automation," IEEE Transactions on Industrial Informatics, 2020, doi: 10.1109/TII.2020.2987629.

[7] M. A. Umer, A. Mathur, K. N. Junejo, and S. Adepu, "Integrating Design and Data Centric Approaches to Generate Invariants for Distributed Attack Detection," in Proceedings of the 2017 Workshop on Cyber-Physical Systems Security and PrivaCy, Nov. 2017, pp. 131-136.

[8] F. Pasqualetti, F. Dörfler, and F. Bullo, "Attack Detection and Identification in Cyber-Physical Systems," IEEE Transactions on automatic control, vol. 58, no. 11, pp. 2715-2729, Nov. 2013.

[9] M. Kravchik, and A. Shabtai, "Detecting Cyber Attacks in Industrial Control Systems Using Convolutional Neural Networks," in Proceedings of CPS-SPC 18 Conference, Oct. 2018, pp. 72-83.

[10] J. Inoue, Y. Yamagata, Y. Chen, C. M. Poskitt, and J. Sun, "Anomaly detection for a water treatment system using unsupervised machine learning," in Proceedings of IEEE International Conference on Data Mining, Nov. 2017, pp. 1058-1065.

[11] D. Hadžiosmanović, R. Sommer, E. Zambon, and P. H. Hartel, "Through the eye of the PLC: semantic security monitoring for industrial processes," in Proceedings of 30th Annual Computer Security Applications Conference, Dec. 2014, pp. 126-135.

[12] Centre for Research in Cyber Security, Singapore University of Technology and Design, "Secure Water Treatment (SWaT)," [Online], Available: http://itrust.sutd.edu.sg/research/testbeds/secure-water-treatmentswat/, Accessed on: May 25, 2020.

[13] D. M. Nedeljkovic, Z. B. Jakovljevic, Z. Dj. Miljkovic, and M. Pajic, "Detection of cyber-attacks in electro-pneumatic positioning system with distributed control," in 27th Telecommunications Forum (TELFOR 2019), Nov. 2019, art. no. 8971062

[14] A. J. Smola, and B. Schölkopf, "A tutorial on support vector regression," Statistics and Computing, vol. 14, no. 3, pp. 199-222, 2004.

[15] V. Vapnik, The Nature of Statistical Learning Theory. Springer, New York, 1995.

[16] J. Goh, S. Adepu, K. N. Junejo, and A. Mathur, “A dataset to support research in the design of secure water treatment systems," in Proceedings of the 11th International Conference on Critical Information Infrastructures Security, Oct. 2016, pp. 88-99.

[17] NXP Semiconductors N. V. (2009, Feb.), "LPC1769/68/66/65/64/63 32-bit ARM Cortex-M3 microcontroller," [Online]. Available: https://www.nxp.com/docs/en/data-

sheet/LPC1769_68_67_66_65_64_63.pdf, Accessed on: May 25, 2020.

[18] Microchip Technology Inc. (2008, Jan.), "MRF24J40MA 2.4 GHz IEEE Std. 802.15.4TM RF Transceiver Module," [Online]. Available:

http://ww1.microchip.com/downloads/en/DeviceDoc/70329b.pdf, Accessed on: May 25, 2020. 